03

\title{
Спектральная и угловая зависимости эффективности трехслойных рельефно-фазовых дифракционных элементов ИК диапазона
}

\author{
(С) Г.И. Грейсух ${ }^{1}$, В.А. Данилов ${ }^{2}$, С.А. Степанов ${ }^{1}$, А.И. Антонов ${ }^{1}$, Б.А. Усиевич ${ }^{3}$ \\ ${ }^{1}$ Пензенский государственный университет архитектуры и строительства, \\ 440028 Пенза, Россия \\ ${ }^{2}$ Научно-технологический центр уникального приборостроения РАН, \\ 117342 Москва, Россия \\ ${ }^{3}$ Институт общей фоизики им. А.М. Прохорова РАН, \\ 119991 Москва, Россия \\ e-mail: grey@pguas.ru
}

Поступила в редакцию 27.02.2018 г.

\begin{abstract}
В рамках скалярной и строгой теории дифракции, основанной на решении системы уравнений Максвелла, проведено исследование пилообразных рельефно-фазовых трехслойных микроструктур, рассчитанных на работу с полихроматическим излучением среднего ИК диапазона $(3 \leq \lambda \leq 5 \mu \mathrm{m})$. Расширен ряд эффективных микроструктур, скомпонованных из различных оптических материалов, и минимизированы глубины их рельефов. Ряд расширен, в частности, за счет микроструктур, у которых френелевские потери на отражение от обоих рельефов не превышают $10 \%$. Этот фактор в совокупности с относительно небольшой суммарной глубиной рельефов может оказаться определяющим при выборе той или иной микроструктуры для решения конкретной научной или технической задачи.
\end{abstract}

DOI: $10.21883 / \mathrm{OS} .2018 .07 .46267 .57-18$

\section{Введение}

Снижение дифракционной эффективности (ДЭ) дифракционных оптических элементов (ДОЭ) при изменении длины волны и угла падения излучения на элемент является одним из наиболее серьезных факторов, сдерживающих использование таких элементов в изображающих, фокусирующих и др. оптических системах.

Спектральная и угловая зависимости эффективности трехслойных рельефно-фазовых дифракционных оптических элементов, рассчитанных на видимое излучение, достаточно хорошо изучены. Так, в работах [1-5] в рамках скалярной теории дифракции (СТД) получены выражения для ДЭ трехслойных пилообразных рельефно-фазовых микроструктур, предложены методы выбора оптических материалов и компоновки таких микроструктур, а также получения оптимальных глубин пилообразных микрорельефов (рис. 1).

Показано, что при соответствующем выборе трех оптических материалов и приемлемых (с практической точки зрения) оптимальных глубинах рельефов в пределах видимого спектрального диапазона $(0.4 \mu \mathrm{m} \leq \lambda \leq$ $\leq 0.7 \mu \mathrm{m})$ и в интервале углов падения излучения из воздуха на микроструктуру $\left(-30^{\circ} \leq \theta \leq 30^{\circ}\right)$ минимальная ДЭ в первом рабочем порядке, полученная в рамках СТД, $\eta_{S, \min } \geq 0.95$. Более того, если допустить, чтобы суммарная глубина рельефов $h_{\Sigma}=h_{1}+h_{2}$ не превышала $37 \mu \mathrm{m}$, то, как показано в [5], можно подобрать комбинацию оптических материалов, обеспечивающих $\eta_{S, \min } \geq 0.95$ в интервале углов падения излучения из воздуха на микроструктуру $-70^{\circ} \leq \theta \leq 70^{\circ}$. К сожалению, полученные в рамках СТД и приведенные выше интервалы углов падения излучения существенно превышают реально достижимые, которые прогнозируются строгой теорией дифракции, основанной на решении системы уравнений Максвелла с соответствующими граничными условиями. Действительно, отношение допустимых углов падения излучения, прогнозируемых СТД - $\theta$ и строгой теорией дифракции - $\Psi$, лежит в диапазоне $1.3 \leq \theta / \Psi \leq 1.7[5]$.

Интерес к использованию ДОЭ в приборах ИК диапазона обусловил исследования, направленные на созда-

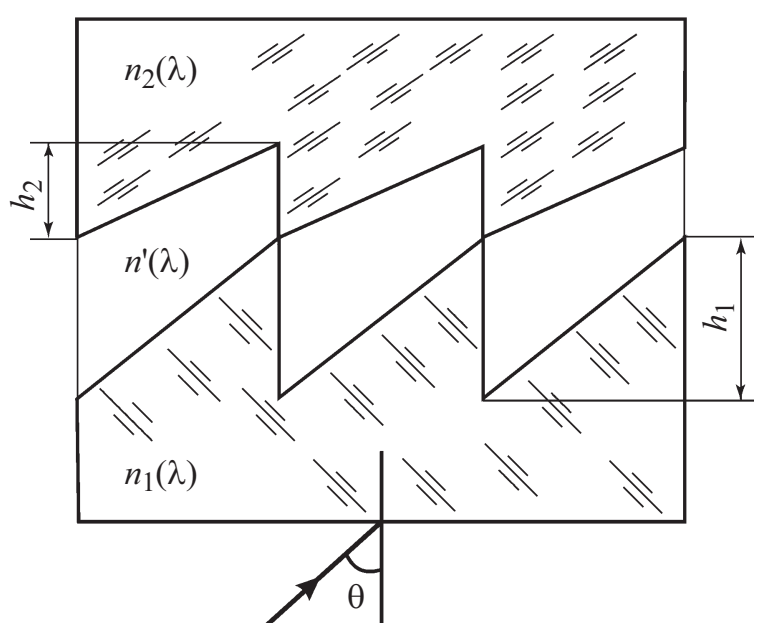

Рис. 1. Трехслойная пилообразная рельефно-фазовая микроструктура. 
ние высокоэффективных дифракционных микроструктур этого диапазона. Так, в работе [6] предложены и в рамках СТД исследованы трехслойные рельефно-фазовые микроструктуры, рассчитанные на полихроматическое излучение среднего ИК диапазона $(3 \leq \lambda \leq 5 \mu \mathrm{m})$. Эти микроструктуры компонуются из обычно используемых в этом диапазоне оптических материалов, таких как $\mathrm{Al}_{2} \mathrm{O}_{3}, \mathrm{Ge}, \mathrm{MgF}_{2}, \mathrm{Si}, \mathrm{SiO}_{2}, \mathrm{ZnS}$. ДЭ микроструктур $\eta_{S}$ в зависимости от длины волны $\lambda$ и угла падения излучения $\theta$ оценивается по формулам, предложенным в работе [1]. При этом глубины рельефов $h_{1}$ и $h_{2}$ получают путем решения системы двух уравнений, обеспечивающих $\eta_{S}=1$ на двух выбранных длинах волн заданного спектрального диапазона при нормальном падении излучения $(\theta=0)$. Показано, что у некоторых микроструктур $\eta_{S, \min }$ больше 0.9 или даже 0.95 при углах падения излучения свыше $65^{\circ}$.

Очевидно, что используемый авторами работы [6] подход к получению глубин рельефов трудно назвать оптимальным, а главное, есть основания сомневаться в том, что полученные в рамках СТД предельно допустимые углы падения излучения соответствуют реально достижимым. Действительно, если явное несоответствие этих углов наблюдается в видимом диапазоне, то в ИК диапазоне соответствие углов (или степень их несоответствия) требует, как минимум, оценки. Более того, только в рамках строгой теории дифракции можно оценить и сопоставить потенциальные возможности каждой из предложенных в [6] трехслойных микроструктур, а следовательно, и прогнозировать перспективы их практического использования.

\section{Используемые методы исследования и критерии}

Для вычисления ДЭ трехслойных микроструктур в рамках СТД за основу также взяты соответствующие формулы работы [1], но с целью объективного сопоставления микроструктур, скомпонованных из различных материалов, в них угол падения излучения в оптическом материале заменен на угол падения излучения из воздуха в материал с показателем преломления $n_{1}(\lambda)$ (рис. 1). В результате формулы приобрели вид

$$
\eta_{S}=\left[\frac{\sin (\pi(m-\chi))}{\pi(m-\chi)}\right]^{2},
$$

где

$$
\begin{aligned}
\chi= & \left(\frac{1}{\lambda}\right)\left[h_{1}\left(\sqrt{n^{\prime 2}-\sin ^{2} \theta}-\sqrt{n_{1}^{2}-\sin ^{2} \theta}\right)\right. \\
& \left.+h_{2}\left(\sqrt{n_{2}^{2}-\sin ^{2} \theta}-\sqrt{n^{\prime 2}-\sin ^{2} \theta}\right)\right]
\end{aligned}
$$

$m$ - номер порядка дифракции.

Оптимальные значения глубин рельефов $h_{1}$ и $h_{2}$ для выбранной тройки оптических материалов зависят от выбора критерия оптимальности. Если предполагается использовать ДОЭ в спектральном приборе или изображающей оптической системе и дифракция излучения в побочные порядки нежелательна на любой длине волны рабочего спектрального диапазона, то наиболее адекватным является критерий, предложенный в работе [7]. В соответствии с ним за оптимальные значения глубин $h_{1}$ и $h_{2}$ принимаются значения, обеспечивающие в выбранном спектральном диапазоне максимально возможный интервал углов падения излучения, в пределах которого ДЭ (в точке ее минимума) не опускалась ниже заданного уровня.

Для оценки ДЭ трехслойной микроструктуры в рамках строгой теории дифракции, основанной на решении системы уравнений Максвелла, используется строгий метод связанных волн (Rigorous coupled-wave analysis (RCWA)) [8]. В соответствии с ним пилообразные рельефы дифракционной микроструктуры заменяются ступенчатыми, и каждая ступень ограничивает тонкий слой, образующий бинарную диэлектрическую решетку, к которой применяется разложение в ряд Фурье. Наши исследования, выполненные RCWA-методом с использованием компьютерной программы, представленной в [9], показали, что в случае трехслойной микроструктуры хорошая сходимость результатов расчета, а следовательно, и их высокая достоверность достигаются при высоте ступеней порядка $(0.15-0.2) \lambda_{\min }$ и числе гармоник ряда Фурье больше 300. Здесь $\lambda_{\min }-$ минимальная длина волны рабочего спектрального диапазона.

Для сопоставления углов падения излучения на микроструктуру, приводящих к снижению ДЭ до одного и того же уровня, но полученных двумя различными методами, эти углы обозначены разными буквами: $\theta-$ в рамках СТД и $\Psi$ - RCWA-методом. Это вызвано тем, что в силу асимметрии рельефа модуль отрицательного угла падения $\left|\Psi_{N}\right|$ и положительный угол падения излучения $\Psi_{P}$, приводящие к снижению ДЭ, оцениваемого RCWA-методом, до одного и того же уровня, могут существенно отличаться, и за оценочный угол $\Psi$, как и в работах $[5,7]$, в настоящей работе принят наименьший из углов $\left|\Psi_{N}\right|$ и $\Psi_{P}$ падения излучения из воздуха на ДОЭ, приводящий к снижению ДЭ до заданного уровня.

Завершая раздел, заметим, что при расчете ДЭ RCWA-методом (в отличие от расчета в рамках СТД) учитываются френелевские потери, обусловленные отражением излучения от обоих рельефов, т.е. оценивается фактическая эффективность микроструктуры, обозначенная ниже как $\eta_{E M}$. В то же время, чтобы обеспечить корректность сравнения ДЭ, полученных разными методами, френелевские потери исключаются путем нормирования значений ДЭ, полученных RCWA-методом, на максимальное значение ДЭ в пределах выбранного спектрального диапазона, но полученное при нормальном падении излучения на ДОЭ $(\Psi=0)$. Нормированная ДЭ обозначена как $\eta_{E M}^{(N)}$. Все расчеты, как и в работе [6], выполнялись в среднем ИК диапазоне $(3 \leq \lambda \leq 5 \mu \mathrm{m})$. 
Конструктивные параметры, дифракционная эффективность и френелевские потери трехслойных микроструктур

\begin{tabular}{|c|c|c|c|c|c|c|c|c|c|}
\hline № & Оптические материалы & Источник & $h_{1}, \mu \mathrm{m}$ & $h_{2}, \mu \mathrm{m}$ & $\theta_{\max }, \operatorname{grad}$ & $\eta_{S, \min }$ & $\eta_{E M, \text { min }}$ & $\eta_{E M, \text { min }}^{(N)}$ & Френелевские потери, \% \\
\hline \multirow{4}{*}{1} & \multirow{4}{*}{$\mathrm{MgF}_{2} / \mathrm{SiO}_{2} / \mathrm{Si}[6]$} & \multirow{2}{*}[\mathrm{HC}]{} & \multirow{2}{*}{-23.1} & \multirow{2}{*}{2.10} & \multirow{2}{*}{80} & \multirow{2}{*}{0.94} & 0.71 & 0.87 & \multirow{4}{*}{17.9} \\
\hline & & & & & & & при $\Psi_{\mathrm{n}}$ & $=30^{\circ}$ & \\
\hline & & \multirow{2}{*}[6]{} & \multirow{2}{*}{-27.84} & \multirow{2}{*}{2.46} & \multirow{2}{*}{25} & \multirow{2}{*}{0.94} & 0.61 & 0.75 & \\
\hline & & & & & & & при $\Psi_{\mathrm{n}}$ & $=30^{\circ}$ & \\
\hline \multirow{4}{*}{2} & \multirow{4}{*}{$\mathrm{SiO}_{2} / \mathrm{ZnS} / \mathrm{Al}_{2} \mathrm{O}_{3}[6]$} & \multirow{2}{*}[\mathrm{HC}]{} & \multirow{2}{*}{-13} & \multirow{2}{*}{-26.20} & \multirow{2}{*}{65} & \multirow{2}{*}{0.95} & 0.74 & 0.82 & \multirow{4}{*}{7.7} \\
\hline & & & & & & & при $\Psi_{\mathrm{n}}$ & $=30^{\circ}$ & \\
\hline & & \multirow{2}{*}[6]{} & \multirow{2}{*}{-31.06} & \multirow{2}{*}{-53.47} & \multirow{2}{*}{25} & \multirow{2}{*}{0.47} & 0.32 & 0.35 & \\
\hline & & & & & & & при $\Psi_{\mathrm{n}}$ & $=20^{\circ}$ & \\
\hline \multirow{4}{*}{3} & \multirow{4}{*}{$\mathrm{MgF}_{2} / \mathrm{SiO}_{2} / \mathrm{Al}_{2} \mathrm{O}_{3}[6]$} & \multirow{2}{*}[\mathrm{HC}]{} & \multirow{2}{*}{-31.77} & \multirow{2}{*}{16.56} & \multirow{2}{*}{40} & \multirow{2}{*}{0.81} & 0.75 & 0.76 & \\
\hline & & & & & & & при $\Psi_{\mathrm{n}}$ & $=20^{\circ}$ & 08 \\
\hline & & {$[6]$} & -3107 & 1806 & 40 & 0.53 & 0.52 & 0.53 & \\
\hline & & {$[0]$} & -51.91 & 10.00 & 40 & 0.53 & при $\Psi_{\mathrm{n}}$ & $=20^{\circ}$ & \\
\hline 4 & $7 n \mathrm{~S} / \mathrm{Si} / \mathrm{Ge}[6]$ & {$[\mathrm{HC}]$} & $-18 \quad 36$ & 2074 & 30 & 081 & 0.38 & 0.40 & 40 \\
\hline 4 & ZnS/SI/Ue $[0]$ & [HC] & -18.50 & 29.14 & 30 & 0.81 & при $\Psi_{\mathrm{n}}$ & $=20^{\circ}$ & 4.9 \\
\hline & & & & & & & 0.71 & 0.80 & \\
\hline 5 & $\mathrm{Al}_{2} \mathrm{O}_{2} / \mathrm{ZnS} / \mathrm{MgF}_{2}$ & {$[\mathrm{HC}]$} & -14.15 & -508 & 80 & 0.91 & при $\Psi_{\mathrm{n}}$ & $=30^{\circ}$ & 83 \\
\hline & & & & & & & 0.75 & 0.84 & \\
\hline & & & & & & & при $\Psi_{\mathrm{n}}$ & $=20^{\circ}$ & \\
\hline 6 & $\mathrm{Al}_{2} \mathrm{O}_{2} / \mathrm{MoF}_{2} / \mathrm{AMTIR}_{3}$ & {$[\mathrm{HCl}$} & -1396 & -666 & 80 & 097 & 0.68 & 0.79 & 111 \\
\hline & & & & & & & при $\Psi_{\mathrm{n}}$ & $=30^{\circ}$ & 11.1 \\
\hline 7 & Alo $2 / 7 n$ S/AMTIR 3 & {$[\mathrm{HC}]$} & -1144 & 742 & 40 & 003 & 0.68 & 0.72 & 28 \\
\hline 1 & $\mathrm{Al}_{2} \mathrm{O}_{3} / \mathrm{LnS} / \mathrm{AIMTIK} 3$ & {$[\mathrm{HC}]$} & & 1.42 & 40 & & при $\Psi$ & $=30^{\circ}$ & 2.8 \\
\hline
\end{tabular}

\section{Результаты исследований}

Основные результаты наших исследований трехслойных микроструктур, как в рамках СТД, так и RCWAметодом, сведены в таблицу. В ней в столбце „Оптические материалы“ для заимствованных конфигураций указана работа [6], в которой данная конфигурация предложена впервые. В столбце „Источник“ указаны либо работа [6], либо настоящая работа [HC], в которых получены представленные в соответствующих столбцах глубины рельефов $h_{1}$ и $h_{2}$. Знак глубин, как и в работе [6], обеспечивает максимальную ДЭ в первом порядке дифракции $(m=1)$.

В столбце $\theta_{\max }$ указан интервал углов $\left(-\theta_{\max } \leq \theta \leq\right.$ $\left.\leq \theta_{\max }\right)$, в пределах которых ДЭ, рассчитанная в рамках СТД, в выбранном спектральном диапазоне не опускается ниже величины, приведенной в столбце $\eta_{S, \min }$. В столбцах $\eta_{E M, \min }$ и $\eta_{E M, \min }^{(N)}$ приведены значения, полученные RCWA-методом, ниже которых ДЭ не опускается в выбранном спектральном диапазоне и в интервале углов $-\Psi_{\max } \leq \Psi \leq \Psi_{\max }$. Эти значения ДЭ получены для микроструктуры с пространственным периодом, десятикратно превышающим суммарную глубину пилообразных рельефов, $\Lambda=10\left(\left|h_{1}\right|+\left|h_{2}\right|\right)$ [7].

Как следует из таблицы, переход при определении оптимальных глубин рельефов от решения системы двух уравнений к использованию критерия, предложенного в работе [7], дает несомненный выигрыш, наблюдаемый по результатам расчетов как в рамках СТД, так и RCWA-методом. Но главное, что использование критерия, предложенного в работе [7], позволило раскрыть потенциальные возможности конфигураций, описанных в работе [6], и минимизировать глубины их рельефов. Кроме того, это позволило предложить новые эффективные конфигурации, компонуя их из материалов, использованных в работе [6], и материала AMTIR3 из каталога „INFRARED“ программы оптического проектирования ZEMAX. Сопоставляя результаты расчетов, выполненных RCWA-методом и представленных в столбцах $\eta_{E M, \min }^{(N)}$ и $\eta_{E M, \min }$, нетрудно видеть, что наилучшей 


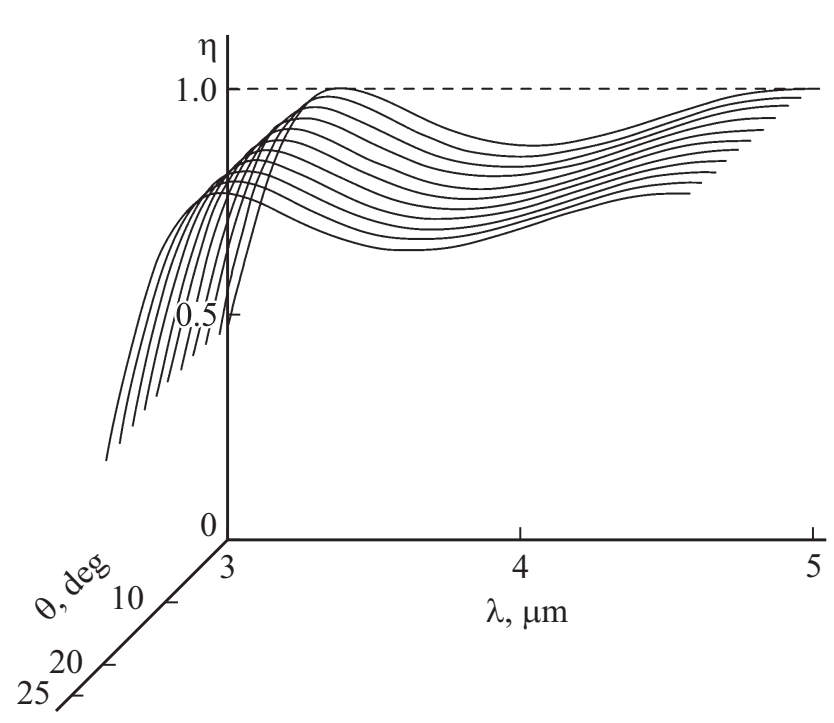

Рис. 2. Зависимость ДЭ, полученная в рамках СТД, от длины волны и угла падения излучения на микроструктуру в конфигурации № $2\left(\mathrm{SiO}_{2} / \mathrm{ZnS} / \mathrm{Al}_{2} \mathrm{O}_{3}\right)$ при глубинах рельефов $h_{1}=-31.06 \mu \mathrm{m}$ и $h_{2}=-53.47 \mu \mathrm{m}$.

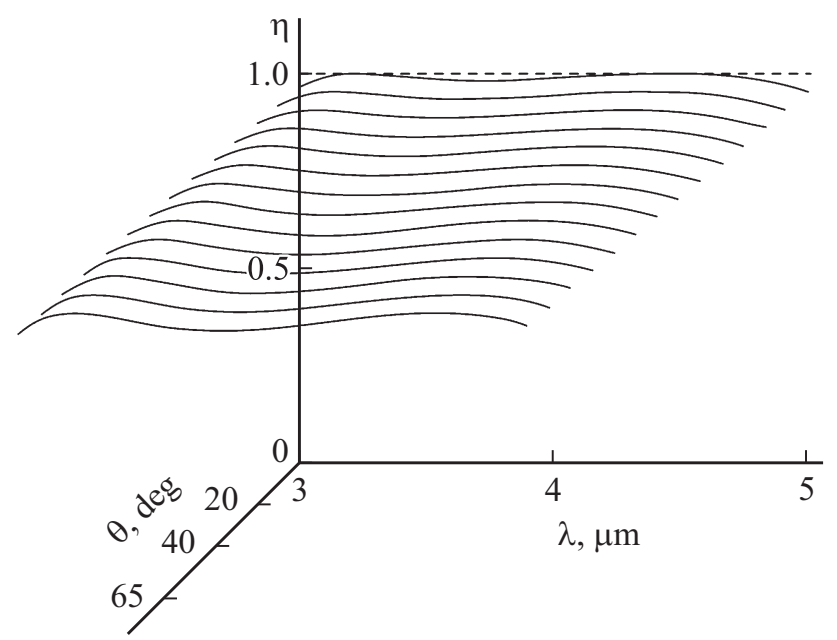

Рис. 3. Зависимость ДЭ, полученная в рамках СТД, от длины волны и угла падения излучения на микроструктуру в конфигурации № $2\left(\mathrm{SiO}_{2} / \mathrm{ZnS} / \mathrm{Al}_{2} \mathrm{O}_{3}\right)$ при глубинах рельефов $h_{1}=-13 \mu \mathrm{m}$ и $h_{2}=-26.20 \mu \mathrm{m}$.

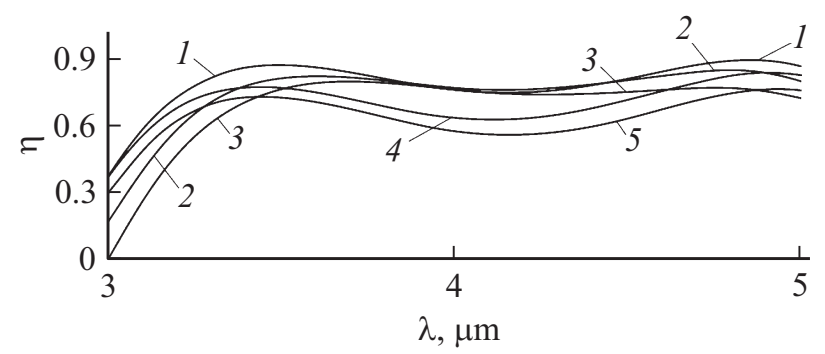

Рис. 4. Зависимости ДЭ от длины волны, полученные RCWA-методом, для микроструктуры в конфигурации № 2 $\left(\mathrm{SiO}_{2} / \mathrm{ZnS} / \mathrm{Al}_{2} \mathrm{O}_{3}\right)$ при глубинах рельефов $h_{1}=-31.06 \mu \mathrm{m}$ и $h_{2}=-53.47 \mu \mathrm{m}$. $\Psi=0^{\circ}(1) ;-20^{\circ}(2) ;-30^{\circ}$ (3); $20^{\circ}$ (4); $30^{\circ}(5)$.

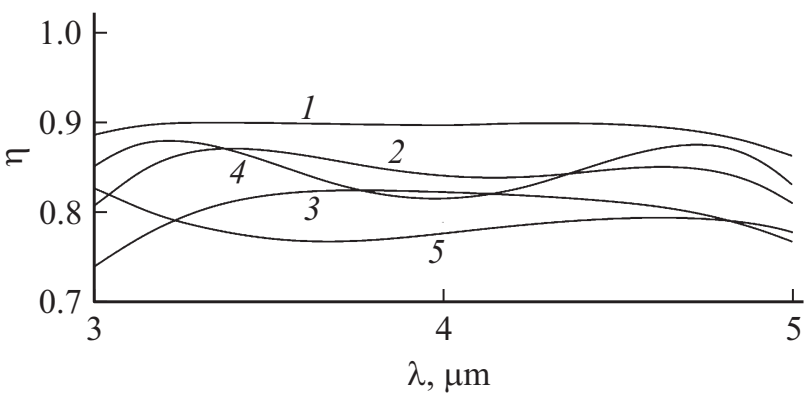

Рис. 5. Зависимости ДЭ от длины волны, полученные RCWA-методом, для микроструктуры в конфигурации № 2 $\left(\mathrm{SiO}_{2} / \mathrm{ZnS} / \mathrm{Al}_{2} \mathrm{O}_{3}\right)$ при глубинах рельефов $h_{1}=-13 \mu \mathrm{m}$ и $h_{2}=-26.20 \mu \mathrm{m} . \Psi=0^{\circ}(1) ;-20^{\circ}(2) ;-30^{\circ}(3) ; 20^{\circ}$ (4); $30^{\circ}(5)$.

конфигурацией как с точки зрения нормированной ДЭ, не учитывающей френелевские потери, так и с точки зрения фактической ДЭ является конфигурация № 2 $\left(\mathrm{SiO}_{2} / \mathrm{ZnS} / \mathrm{Al}_{2} \mathrm{O}_{3}\right)$ при глубинах рельефов $h_{1}=-13 \mu \mathrm{m}$ и $h_{2}=-26.20 \mu \mathrm{m}$. На рис. 2-5 для этой конфигурации приведен ряд графиков, более наглядно иллюстрирующих выигрыш, достигнутый благодаря оптимизации глубин обоих рельефов микроструктуры.

Что касается конфигураций № 5-7, то они по параметрам $\eta_{E M, \min }^{(N)}$ и $\eta_{S, \min }$ несколько уступают конфигурации № 2, но зато отличаются значительно меньшими глубинами рельефов. Это может играть определяющую роль с точки зрения технологии изготовления микроструктур.

\section{Заключение}

В настоящей работе в рамках скалярной и строгой теории дифракции (RCWA-методом) проведено исследование пилообразных рельефно-фазовых трехслойных микроструктур, рассчитанных на работу с полихроматическим излучением среднего ИК диапазона $(3 \leq \lambda \leq 5 \mu \mathrm{m})$. При этом в качестве критерия оценки эффективности микроструктуры использовался критерий, предложенный в работе [7] и требующий, чтобы во всем рабочем спектральном диапазоне обеспечивался максимально возможный интервал углов падения излучения, в пределах которого ДЭ (в точке ее минимума) не опускается ниже заданного уровня.

Данный критерий позволил не только оптимизировать глубины рельефов микроструктур в уже известных конфигурациях, но и организовать в рамках СТД из предложенного каталога оптических материалов автоматический процесс компоновки новых конфигураций, обеспечивающих выполнение заданных требований при минимально возможной суммарной глубине рельефов.

Были сопоставлены значения ДЭ, прогнозируемые CTД и RCWA-методом, и в результате были раскрыты потенциальные возможности конфигураций, описанных 
в работе [6], и минимизированы глубины их рельефов. Кроме того, были предложены новые эффективные конфигурации, скомпонованные из материалов, использованных в работе [6], и материала AMTIR3 из каталога „INFRARED“ программы оптического проектирования ZEMAX. При этом новые конфигурации пополнили ряд тех, у которых френелевские потери на отражение от обоих рельефов не превышают $10 \%$. Этот фактор в совокупности с относительно небольшой суммарной глубиной рельефов может оказаться определяющим при выборе трехслойной микроструктуры в той или иной конфигурации для решения конкретной научной или технической задачи.

Работа выполнена при финансовой поддержке Российского научного фонда (проект № 17-19-01461).

\section{Список литературы}

[1] Zhao Y.H., Fan C.J, Ying C.F., Liu S.H. // Opt. Commun. 2013. V. 295. P. 104.

[2] Shan Mao, Qingfeng Cui, Mingxu Piao, Lidong Zhao /I Appl. Optics. 2016. V. 55. N 13. P. 3549-3554.

[3] Ya-hui Zhao, Chang-jiang Fan, Chao-fu Ying, Hui Wang /I Optik. 2013. V. 124. N 20. P. 4142-4144.

[4] Fan Changjiang // Proc. SPIE. V. 9272. N 92720. P. 1-6.

[5] Грейсух Г.И., Данилов В.А., Ежов Е.Г., Степанов С.А., Усиевич Б.А. // Опт. и спектр. 2015. Т. 118. № 6. С. 118.

[6] Hongbo Xie, Delun Ren, Chao Wang, Chensheng Mao\&Lei Yang // J. Mod. Optics. 2018. V. 65. N 3. P. 255-261.

[7] Грейсух Г.И., Данилов В.А., Ежов Е.Г., Степанов С.А., Усиевич Б.А. // Оптический журнал. 2015. Т. 82. № 5. С. 56.

[8] Moharam M.G., Gaylord T.K. // J. Opt. Soc. Am. 1982. V. 72. N 10. P. 1385.

[9] Lyndin N.M. Modal and C Methods Grating Design and Analysis Software. [Электронный ресурс] Режим доступа: http://www.mcgrating.com 